\title{
Pyruvate dehydrogenase E3 deficiency
}

INSERM

\section{Source}

INSERM. (1999). Orphanet: an online rare disease and orphan drug data base. Pyruvate dehydrogenase E3 deficiency. ORPHA:2394

Pyruvate dehydrogenase E3 deficiency is a very rare subtype of pyruvate dehydrogenase deficiency (PDHD, see this term) characterized by either early-onset lactic acidosis and delayed development, later-onset neurological dysfunction or liver disease. 